TRANSACTIONS OF THE

AMERICAN MATHEMATICAL SOCIETY

Volume 197, 1974

\title{
SYMPLECTIC HOMOGENEOUS SPACES
}

BY

\author{
BON-YAO CHU (1)
}

ABSTRACT. It is proved in this paper that for a given simply connected Lie group $G$ with Lie algebra 8 , every left-invariant closed 2-form induces a symplectic homogeneous space. This fact generalizes the results in [7] and [12] that if $H^{1}(g)=H^{2}(g)=0$, then the most general symplectic homogeneous space covers an orbit in the dual of the Lie algebra g. A one-to-one correspondence can be established between the orbit space of equivalent classes of 2-cocycles of a given Lie algebra and the set of equivalent classes of simply connected symplectic homogeneous spaces of the Lie group. Lie groups with left-invariant symplectic structure cannot be semisimple; hence such groups of dimension four have to be solvable, and connected unimodular groups with left-invariant symplectic structure are solvable [4].

1. Symplectic manifolds. Let $M$ be a $2 n$-dimensional connected differentiable manifold. A symplectic structure on $M$ is defined by a closed differential 2form $\omega$ which is everywhere of maximal rank. Such a form is called a symplectic form of the symplectic structure defined on $M$. On a symplectic manifold $M$, a one-to-one map from the space of vector fields $X(M)$ onto the space of linear differential forms $D^{1}(M)$ can be defined as follows. If $X$ is a vector field, the map $x \mapsto i(X)_{\omega}$ (where $i(X)_{\omega}$ denotes the interior product of $X$ with $\omega$ ) is a bijective map from $X(M)$ onto $D^{1}(M)$. In fact, at each point $x \in M$, this map from $T_{\boldsymbol{x}}(M)$ onto $T_{x}^{*}(M)$ is given by the nonsingular bilinear form $\omega_{x}$ A classical theorem attributed to Darboux [11] states that for an $n$-dimensional manifold $M$ with a closed 2-form $\omega$ of rank exactly $p$ everywhere there can be introduced about every point a system of coordinates $x^{1}, \cdots, x^{n-p}, y^{1}, \cdots, y^{p}$, in terms of which the local representation of $\omega$ becomes

Received by the editors April 23, 1973 and, in revised form, July 10, 1973.

AMS (MOS) subject classifications (1970). Primary 53C 30; Secondary 57F 15, 22 E 25.

Key words and phrases. Symplectic structure, homogeneous space, differential form, regular involutive distribution, affine transformation group, cohomology, semisimple, solvable and unimodular Lie groups.

(1) This work is based on a dissertation submitted in partial fulfillment of the requirements for the Ph.D. degree at the University of Notre Dame. The author wishes to thank Professor Yozo Matsushima whose guidance and encouragement made the work of this paper possible. 


$$
\omega=d x^{1} \wedge d y^{1}+\cdots+d x^{p} \wedge d y^{p}
$$

such a system is called a canonical system.

An example of a symplectic manifold is provided by the cotangent bundle $T^{*}(M)$ of any manifold $M$ with the natural symplectic form defined as follows. On $T^{*}(M)$ there is a linear form $\theta$ such that, for any tangent vector $X_{u}$ at a point $u$ of $T^{*}(M), \theta_{u}\left(X_{u}\right)=u\left(\pi_{*} X_{u}\right)$, where $\pi$ is the projection of $T^{*}(M)$ onto $M$. The form $\omega=d \theta$ is of maximal rank and therefore defines a symplectic structure on $T^{*}(M)$.

2. Affine transformation groups. Let $\mathbf{R}^{n}$ be the vector space of $n$-tuple of real numbers. We denote it by $A^{n}$ when we regard $\mathrm{R}^{n}$ as an affine space. If $A^{n}$ is considered as a differentiable manifold, then the tangent space to it an an point can be identified in a natural way with the space $\mathbf{R}^{n}$. The group $A(n ; \mathbf{R})$ of affine transformations of $A^{n}$ is represented by the group of all matrices of the form

$$
A(n ; \mathbf{R})=\left\{t=\left[\begin{array}{ll}
l, & c \\
0, & 1
\end{array}\right] \in G L(n+1, \mathbf{R})\right\},
$$

where $l \in G L(n ; \mathbf{R})$ and $c$ is a column vector in $\mathbf{R}^{n}$. The group $A(n ; \mathbf{R})$ is a semidirect product of $\mathbf{R}^{n}$ and $G L(n ; \mathbf{R})$. If we denote by $\mathbf{R}^{n}$ the Lie algebra of the vector group $\mathbf{R}^{n}$, then the Lie algebra $a(n ; \mathbf{R})$ of $A(n ; \mathbf{R})$ is the semidirect sum of $g^{l}(n ; \mathbf{R})$ and $\mathbf{R}^{n}$, which means that an infinitesimal affine transformation $T$ of $A^{n}$ can be expressed in matrix form $\left[\begin{array}{ll}L & c \\ 0 & 0\end{array}\right]$ where $L \in g \mathcal{L}(n ; \mathbf{R})$ and $c$ is a column vector in $\mathbf{R}^{n}[6]$. We call $T \in a(n ; \mathbf{R})$ an infinitesimal affine transformation of $A^{n}, L$ the linear part and $c$ the translation part of $T$ respectively. The exponential map of the Lie algebra of infinitesimal affine transformations into the affine transformation group is given by the formula

$$
(\exp T)(x)=(\exp L)(x)+\left(\sum_{n=1}^{\infty} \frac{1}{n !} L^{n-1}\right)(c)
$$

for $x \in A^{n}$.

Let $G$ be a connected semisimple Lie group with Lie algebra $g$, then the first cohomology group $H^{1}(g, \rho, V)$ is trivial for any representation $\rho$ of $g$ by linear transformations on a finite-dimensional vector space $V[3]$. Therefore there exists a one-to-one correspondence between the coboundaries $B^{1}(g, \rho, V)$ and the set of all infinitesimal affine representations of $g$ in $V$ with linear part $\rho$, and it is also evident that a connected semisimple Lie group $G$ operating on a finite-dimensional vector space $V$ as an affine transformation group has a fixed point $\nu_{0} \in V$ under the action of $G$. 
3. Symplectic homogeneous spaces of a given Lie group. Let $G$ be a connected Lie group and $H$ a closed connected subgroup of $G$. Denote by $g$ and $G$ the Lie algebras of $G$ and $H$ respectively, by $M$ the homogeneous space $G / H$, and by $\pi$ the map $g \mapsto g H$ of $G$ onto $M$; consider $G$ as a group of operators for $M$ by setting $t_{g}\left(g^{\prime} H\right)=g g^{\prime} H$. The map $\omega \mapsto \pi^{*} \omega$ establishes a one-to-one correspondence between the invariant forms of order $p$ on $M$ and those left-invariant $p$-forms $\theta$ on $G$ which satisfy the conditions

(i) $i(X) \theta=0$ for all $X \in \mathfrak{h}$,

(ii) $\theta$ invariant under the adjoint map of $H[3]$.

We say a homogeneous space $M=G / H$ is symplectic if $M$ has a symplectic structure and the symplectic form $\omega$ of it is $G$-invariant under the natural action by $t_{g}\left(g^{\prime} H\right)=g g^{\prime} H$. When $M$ has an invariant symplectic structure defined by a closed 2-form $\omega$, the dimension of $M$ is, of course, an even integer $2 p$ and $\omega^{p}$, being nonzero everywhere, defines a volume element of $M$. Denote by $\tilde{\omega}$ the pull back form $\pi^{*} \omega$ on $G$, since $\omega$ is nondegenerate, we see that an element $X$ of $g$ belongs to $\mathscr{G}$ if and only if $\tilde{\omega}(X, Y)=0$ for all $Y \in \mathrm{g}$.

The purpose of this section is to establish a correspondence between the space $Z^{2}(g)$ of 2 -cocycles of $g$ and the set of all symplectic homogeneous spaces of $G$.

Let $M$ be any $n$-dimensional differentiable manifold. An $m$-dimensional involutive distribution $D$ on $M$ is said to be regular, if every point $x \in M$ possesses a neighborhood $U_{\boldsymbol{x}}$ in $M$ such that each maximal connected integral submanifold of $D$ intersects $U_{x}$ in at most one $m$-dimensional slice [9]. If $\omega$ is a closed 2form having rank $m$ everywhere, let

$$
\left(D_{\omega}\right)_{x}=\left\{X_{x} \in T_{x}(M) \mid i(X) \omega_{x}=0\right\} .
$$

Then $D_{\omega}$ defines a differentiable involutive distribution on $M_{0} \omega$ is said to be regular if $D_{\omega}$ is regular. The Lie algebra $\mathscr{G}$ corresponding to a closed connected Lie subgroup $H$ of $G$ may be considered as an involutive distribution on $G$, and $H$ is the maximal connected integral submanifold of 5 passing through the identity element $e$ [1]. Conversely, suppose $\bar{G}$ is a Lie subalgebra of 9 , and $\bar{G}$ is a regular involutive distribution on $G$, then, according to a theorem given by Palais [9], every leaf of $G$ is a closed submanifold of $G$. Therefore, in particular, the maximal connected submanifold $H$ of $\mathscr{G}$ passing through the identity is a closed Lie subgroup of $G$.

Theorem 1. Let $G$ be a connected Lie group of $n$ dimensions.

(i) Every 2p-dimensional symplectic homogeneous space of $G$ induces a left-invariant regular 2-form of rank $p$ on $G$. 
(ii) Every left-invariant regular closed 2-form of rank $p$ induces a $2 p$-dimensional symplectic homogeneous space of $G$.

Proof. (i) Let $H$ be the isotropy subgroup of $G$ such that $G / H$ is the given $2 p$-dimensional homogeneous space with the invariant symplectic form $\omega$. The pull back left-invariant closed 2-form $\tilde{\omega}$ on $G$ defines an involutive-distribution

$$
\xi_{\widetilde{\omega}}=\{X \in g \mid z(X) \tilde{\omega}=0\}
$$

which coincides with the Lie algebra 5 of $H$. Hence the identity component of $H$ is the maximal integral submanifold through $e$ of $5_{\omega}$ There exists a neighborhood $U$ of $e$ in $G$ such that $H \cap U$ consists of at most a single slice. Therefore for each $g \in G, g U$ is the desired neighborhood of $g$. Hence $\bar{g} \widetilde{\omega}$ is a regular involutive distribution of dimension $n-2 p$ and $\tilde{\omega}$ is the left-invariant regular closed 2-form on $G$ of rank $p$.

(ii) Let $\theta$ be a left-invariant regular closed 2 -form of rank $p$ on $G$. Then $\mathfrak{G}_{\theta}=\{X \in g \mid i(X) \theta=0\}$ is a regular involutive distribution. The maximal integral submanifold passing through $e$ is a closed subgroup $H_{\theta}$ with Lie algebra $\zeta_{\theta}$, and every leaf through $g$ of $\xi_{\theta}$ is just a right coset $g H_{\theta}$ of $H_{\theta}$. A homogeneous space $G / H_{\theta}$ of dimension $2 p$ is defined and, furthermore, $L_{X} \theta=d i(X) \theta+i(X) d \theta$ $=0$ for all $X \in \xi_{\theta}$. The form $\theta$ is invariant under the adjoint map of $H_{\theta}$, which can be projected down to an invariant form on $G / H_{\theta}$ defining a symplectic structure.

Hence the problem to find all the symplectic homogeneous spaces of a given Lie group is to find all regular left-invariant closed 2 -forms $\theta$ on $G$. This is essential to show that the connected Lie subgroup $H_{\theta}$ corresponding to the Lie subalgebra $G_{\theta}$ is closed in $G$.

Corollary 1. Every left-invariant 2-form $\omega$ on a connected Lie group of the form $\omega=d \theta$ with $\theta$ left-invariant is regular.

Proof. Since $L_{X} \theta=i(X) d \theta+d i(X) \theta, X$ belongs to $\zeta_{\omega}$ if and only if $L_{x} \theta=0$. Then $H=\left\{h \in G \mid R_{b} \theta=\theta\right\}$ is a closed subgroup of $G$ and has Lie algebra equal to $\mathfrak{G}_{\omega} . H_{\omega}$, being the identity component of $H$, is closed.

Corollary 2. If the second dimension cohomology group $H^{2}(\mathrm{~g})$ of the Lie algebra $g$ for a connected Lie group $G$ is trivial, then every left-invariant closed 2form on $G$ induces a symplectic homogeneous space.

Corollary 3 (Kirillov). Every orbit $\mathrm{Ad}^{*}(G) \theta$ of $\mathrm{g}^{*}$ is of even dimension.

Proof. According to Corollary $1, d \theta$ is regular and induces a skewsymmetric 2-form of maximal rank on $\operatorname{Ad}^{*}(G) \theta$, hence $\mathrm{Ad}^{*}(G) \theta$ is even dimensional. 
However, in general not every left-invariant closed 2 -form on a given connected Lie group is regular, since an irregular 2-form can be given on 3-dimensional torus. Thus, instead of solving the problem of finding all symplectic homogeneous spaces of a given Lie group, we now direct our attention to a slightly different one, namely: For a given Lie algebra $g$, let $\omega$ be a cocycle in $Z^{2}(g)$; does there exist a homogeneous space with a symplectic structure induced by $\omega$ ? We may, of course, take the simply connected Lie group $G$ corresponding to the given Lie algebra $g$ as the transformation group of $M$, but it is still essential to show that the Lie subalgebra given by $\zeta_{\omega}=\left\{X \in g \mid i(X)_{\omega}=0\right\}$ defines a closed . Lie subgroup $H_{\omega}$ in $G$. The following theorem answers the question.

Theorem 2. On a simply connected Lie group, every left-invariant closed 2form is regular.

Proof. Denote by $g^{*}$ the dual space of the Lie algebra $g$ for $G$ and regard $g^{*}$ as an affine space. Let $A\left(g^{*}\right)$ be the group of affine transformations of $\dot{g}^{*}$, and $a\left(g^{*}\right)$ be the Lie algebra of infinitesimal affine transformations of $g^{*}$. If we identify the Lie algebra of the vector group $g^{*}$ to itself, then $a\left(g^{*}\right)$ is a semidirect sum of $g^{I}\left(g^{*}\right)$ and $g^{*}$ where $g^{\mathfrak{l}}\left(g^{*}\right)$ denotes the Lie algebra of linear transformations of $g^{*}$. Let $T_{1}$ and $T_{2}$ be two affine infinitesimal transformations in $a\left(g^{*}\right)$ written as the sum of a linear part and a translation part, $T_{1}=L_{1}+c_{1}$ and $T_{2}=L_{2}+c_{2}$ for $L_{1}$ and $L_{2} \in \mathrm{gl}\left(g^{*}\right), c_{1}$ and $c_{2} \in g^{*}$. The Lie product is

$$
\left[T_{1}, T_{2}\right]=\left[L_{1}, L_{2}\right]+L_{1} c_{2}-L_{2} c_{1} \text {, }
$$

where $\left[L_{1}, L_{2}\right]$ denotes the Lie product in $g^{I}\left(g^{*}\right)$. Noting $\omega \in Z^{2}(g)$ and $i(X)_{\omega}$ $\epsilon g^{*}$ for $X \in g$, we now define an affine representation $f_{*}$ of Lie algebra $g$ on the representation space $g^{*}$ as follows:

$$
\begin{aligned}
f_{*}: & g \rightarrow a\left(g^{*}\right), \\
X & \mapsto-{ }^{t} \operatorname{ad}(X)+i(X) \omega .
\end{aligned}
$$

This means for any $\theta \in g^{*}, f_{*}(X)(\theta)=-{ }^{t} \operatorname{ad}(X)(\theta)+i(X) \omega$. Write $-{ }^{t}$ ad $=\mathrm{ad}^{*}$, then $L_{X}=\operatorname{ad}^{*}(X)$ and since $L_{Y} i(X) \omega=i(Y) L_{X} \omega$ for all $X, Y \in g, f_{*}([X, Y])=$ $\left[f_{*}(X), f_{*}(Y)\right.$ ]. Therefore $f_{*}$ is a Lie algebra homomorphism from $g$ onto $d\left(g^{*}\right)$. Because $G$ is simply connected, the Lie algebra homomorphism $f_{*}$ can be represented by a differential of an analytic homomorphism $f$ of $G$ into $A\left(g^{*}\right)$. Thus by the representation $f$, we may regard $G$ as an affine transformation group on $g^{*}$. And the action of $f$ is given for $X \in g, \theta \in g^{*}$, as

$$
f(\exp X)(\theta)={ }^{t} \mathrm{Ad}^{-1}(\exp X) \theta+\sum_{n=1}^{\infty} \frac{1}{n !}\left(L_{X}\right)^{n-1} i(X) \omega .
$$

The isotropy subgroup $H$ of $G$ at origin of $g^{*}$ is closed. Let $X \in \mathscr{G}_{\omega}$ and 
$\exp t X(t \in \mathbf{R})$ be the one parameter subgroup of $G$ generated by $X$; then $f(\exp t X)(0)=0$. This means $\exp t X$ is a one parameter subgroup in $H$. Conversely, suppose $\exp t X(t \in \mathbf{R})$ is a one parameter subgroup in $H$; then $f(\exp t X)(0)=0$ implies $i(X) \omega=0$ and $X \in \mathscr{K}_{\omega}$. Therefore the isotropy subgroup $H$ of $G$ at the origin of $g^{*}$ has the Lie subalgebra $\xi_{\omega} \cdot H_{\omega}$, being the identity component of the Lie group $H$ corresponding to the Lie algebra $\hbar_{\omega}$, is thus closed in $G$, and since $\mathscr{G}_{\omega}$ is regular so is $\omega$.

Let us denote by $\operatorname{Ad}^{*}(G)$ the adjoint representation acting on $g^{*}$ and by $\Omega(\theta)$ the orbit of $\theta$ in $g^{*}$. Suppose, in the above theorem, that $\omega=d \theta$ where $\theta$ is a linear left-invariant form, then

$$
f(\exp X)(0)=\operatorname{Ad}^{*}(\exp X) \theta-\theta \text {. }
$$

The exponential map from $g$ to $G$ is not onto, but it is a local homeomorphism in a neighborhood of the identity. And, since any neighborhood of the identity generates the whole group, we see that

$$
f(g)(0)=\operatorname{Ad}^{*}(g) \theta-\theta \text { for } g \in G .
$$

Let $t_{\theta} \in A\left(g^{*}\right)$ be the translation by $\theta$ carrying each $\phi \in g^{*}$ to $\phi+\theta ; t_{\theta}(\phi)=$ $\phi+\theta$. Replacing $f(g)$ by $t_{\theta} f(g) t_{-} \theta$, we have

$$
t_{\theta} f(g) t_{-\theta}(\theta)=\operatorname{Ad}^{*}(g) \theta \text {. }
$$

Hence every orbit $\Omega(\theta)$ of $G$ in $g^{*}$ is a symplectic homogeneous space and the symplectic structure is induced by the exterior differential of $\theta$. Moreover, we have the following.

Corollary 1. If the second dimension cohomology group $H^{2}(\mathrm{~g})$ of a simply connected Lie group is trivial, then every simply connected symplectic homogeneous space is a covering space of some orbit of $G$ in $g^{*}$.

This result was obtained by Kostant [7] and Souriau [12] in different ways. Kostant considered strongly symplectic homogeneous spaces and proved that there is a one-to-one correspondence between the set of all isomorphism classes of simply connected Hamiltonian spaces and all orbits in $9^{*}$. And Souriau discussed the affine action induced by a cocycle in a slightly different context from the above.

The following is also an easy consequence of previous theorems.

Theorem 3. Let $G$ be a simply connected Lie group with Lie algebra g. Denote by $\mathrm{Sp}(G)$ the set of all simply connected symplectic homogeneous spaces of G. There is a map from $Z^{2}(\mathrm{~g})$ onto $\mathrm{Sp}(G)$ in such a way that:

(i) For $\omega \in Z^{2}(\mathrm{~g})$, there is $M=G^{\prime} H_{\omega} \in \mathrm{Sp}(G)$ where $H_{\omega}$ is the 
connected Lie subgroup of $G$ corresponding to the Lie algebra $\xi_{\omega}$.

(ii) Conversely, for any $M=G / H \in \mathrm{Sp}(G)$, there is $\omega \in Z^{2}(\mathrm{~g})$ such that the isotropy subgroup $H$, under the action of $G$ on $M$, is $H_{\omega}$.

We will give a classification of $\operatorname{Sp}(G)$, but at first let us observe the following lemma.

Lemma. Let $\omega$ be a left-invariant closed 2-form of rank $p$ on $G$. Then for any $X \in g, i(X) \omega=0$ if and only if $i(X) \omega^{p}=0$.

Proof. Since $\omega$ is a closed 2-form of rank $p$ on $G$, by the Darboux Theorem, there is a canonical coordinate system $x^{1}, \ldots, x^{p}, y^{1}, \cdots, y^{p}, z^{1}, \cdots, z^{n-2 p}$ $(n=\operatorname{dim} G$ ) defined around the identity $e$ such that

$$
\omega_{e}=d x_{e}^{1} \wedge d y_{e}^{1}+\cdots+d x_{e}^{p} \wedge d y_{e}^{p}
$$

Let $X \in g$ be expressed in terms of the coordinates at $e$,

$$
X_{e}=\sum_{i=1}^{p} a_{i}\left(\frac{\partial}{\partial x^{i}}\right)_{e}+\sum_{j=1}^{p} b_{j}\left(\frac{\partial}{\partial y^{j}}\right)_{e}+\sum_{k=1}^{n-2 p} c_{k}\left(\frac{\partial}{\partial z^{k}}\right)_{e},
$$

where $a_{i}^{\prime} s, b_{j}$ 's and $c_{j}$ 's are real numbers. Hence $i\left(X_{e}\right) \omega_{e}^{p}=0$ implies $a_{i}=b_{j}$ $=0$ by virtue of the fact that

$$
d x_{e}^{1} \wedge d y_{e}^{1} \wedge \ldots \wedge d \hat{x}_{e}^{i} \wedge d y_{e}^{i} \wedge \ldots \wedge d x_{e}^{p} \wedge d y_{e}^{p}
$$

and

$$
d x_{e}^{1} \wedge d y_{e}^{1} \wedge \ldots \wedge d x_{e}^{j} \wedge d \hat{y}_{e}^{j} \wedge \ldots \wedge d x_{e}^{p} \wedge d y_{e}^{p}
$$

are linearly independent in $T_{e}^{*}(G)$ for $1 \leq i, j \leq p$. Therefore we have $i(X)_{\omega}=0$. The sufficient condition is trivial.

For $M=G^{\prime} H \in \operatorname{Sp}(G)$, let $\pi$ be the natural map from $G$ to $G / H$. The isotropy subgroup of $G$ at the point $\pi(g)=g H$ of $G / H$ is equal to $g H^{-1}$. Hence there is an equivalence relation in $\mathrm{Sp}(G)$, namely, two simply connected symplectic homogeneous spaces $G / H$ and $G / H^{\prime}$ are equivalent if there exists $g \in G$ such that $H^{\prime}=g \mathrm{Hg}^{-1}$. Denote by $\mathrm{Sp}[G]$ the equivalence classes of $\mathrm{Sp}(G)$. An equivalence relation in $Z^{2}(\mathrm{~g})$ can also be defined as follows. Two elements $\omega$ and $\tilde{\omega} \in Z^{2}(g)$ are equivalent if both $\omega$ and $\tilde{\omega}$ are of rank $p$ and $\omega^{p}=c \cdot \tilde{\omega}^{p}$ for some constant $c \neq 0$. Denote by $\mathrm{Sp}[g]$ the equivalence classes in $Z^{2}(g)$. We now have the classification.

Theorem 4. Let $G$ be a simply connected Lie group with Lie algebra g. Let $\operatorname{Ad}(G)$ act on $\mathrm{Sp}[\mathrm{g}]$. Then there is a one-to-one correspondence between the orbit spaces of $\mathrm{Sp}[\mathrm{g}]$ and equivalence classes of $\mathrm{Sp}[G]$ defined as follows. 
For $\omega \in Z^{2}(g)$, let $G / H_{\omega}$ be the corresponding simply connected symplectic homogeneous space in $\mathrm{Sp}(G)$, then the orbit space $\left\{\mathrm{Ad}(G)_{\omega}\right\}$ in $\mathrm{Sp}[g]$ induces the equivalence class $\left[G / g_{\omega} \mathrm{H}^{-1} \mid g \in G\right]$ in $\mathrm{Sp}[G]$.

Proof. Suppose there are symplectic structures $\omega$ and $\tilde{\omega}$ defined on $G / H \in \operatorname{Sp}(G)$ and the dimension of $G / H$ is $2 p$. Both $\omega^{p}$ and $\tilde{\omega}^{p}$ are invariant volume elements on $G / H$, so there is a constant $c \neq 0$ such that $\omega^{p}=c \cdot \tilde{\omega}^{p}$. Denote the pull back forms on $G$ of $\omega$ and $\tilde{\omega}$ by the same letters respectively. We see $\omega^{p}=c \cdot \tilde{\omega}^{p}$ on $G$ and $H=H_{\omega} \cdot{ }^{\prime}$ Hence $G / H \epsilon$ $\mathrm{Sp}(G)$ induces $[\omega] \in \mathrm{Sp}[g]$. Conversely, suppose $\omega$ and $\tilde{\omega}$ in $Z^{2}(g)$ are equivalent. We would like to see $G / H_{\omega}=G / H \tilde{\omega}$. Since $G$ is simply connected, $H_{\omega}$ and $H \widetilde{\omega}$ are connected subgroups of $G$. The only thing to be verified is $\xi_{\omega}=\hbar \tilde{\omega}$ which follows easily from the lemma. Hence $H_{\omega}=H_{\tilde{\omega}}$ and $G / H_{\omega}=G / H_{\omega}^{\sim}$. Therefore we achieve a one-to-one correspondence between $\operatorname{Sp}[g]$ and $\operatorname{Sp}[G]$. Since $\operatorname{Ad}(G)[\omega]=[\operatorname{Ad}(G) \omega]$, the action $\operatorname{Ad}(G)$ on $\mathrm{Sp}(\mathrm{g})$ is well defined. Therefore, the fact that if $\omega \in Z^{2}(\mathrm{~g})$ induces $G / H \epsilon$ $\mathrm{Sp}(G)$, then $\operatorname{Ad}(g) \omega$ induces $G / g H_{\omega} g^{-1} \in \operatorname{Sp}(G)$, establishes the one-to-one correspondence between the orbit space $\operatorname{Ad}(G)[\omega]$ of $\mathrm{Sp}(\mathrm{g})$ and equivalence class $\left[G / g H_{\omega} g^{-1} \mid g \in G\right]$ of $\mathrm{Sp}[G]$.

4. Lie groups with left-invariant symplectic structures. A Lie group is said to have a left-invariant symplectic structure if it has a left-invariant closed 2-form of maximal rank. We call such Lie groups symplectic groups. If $\omega$ is a left-invariant symplectic form on $G$, then $\omega_{e}$ defines a skewsymmetric bilinear function of maximal rank on the Lie algebra $g$ of $G$, and conversely any maximal rank 2 -cocycle of $g$ defines a left-invariant symplectic structure on $G$.

As an example, the cotangent bundle $T^{*}(G)$ of any Lie group $G$ has a natural symplectic structure. We would like to see what properties $G$ will have if the natural symplectic structure is left-invariant under the left translation induced by the elements of Lie group $T^{*}(G)$.

Theorem 5. The natural symplectic structure $\omega$ defined on the cotangent bundle $T^{*}(G)$ of a Lie group $G$ is invariant under the left translations induced by the elements of Lie group $T^{*}(G)$ if and only if $G$ is abelian.

Proof. Let $g$ be the Lie algebra of left-invariant vector fields on $G$ and let $9^{*}$ be the dual space of $g$ consisting of left-invariant 1 -forms on $G$. Every element of $T^{*}(G)$ can be expressed uniquely by the linear function $\alpha_{g}$ of $T_{g}^{*}(G)$ obtained by a left-invariant 1 -form $a$ at $g \in G$. The multiplication in $T^{*}(G){ }^{8}$ is $\alpha_{g} \cdot \beta_{b}=\left(\alpha+{ }^{t} \operatorname{Ad}\left(g^{-1}\right) \beta\right)_{g h}$ and the identity element of $T^{*}(G)$ is $0_{e}$ 
where 0 is the zero form on $G$ and $e$ is the identity of $G$. Let $L_{a_{g}}$ and $L_{g}$ be the left translation induced by $\alpha_{g}$ and $g$ respectively in $T^{*}(G)$ and $G$. Denote by $\pi$ the natural projection $T^{*}(G) \stackrel{\boldsymbol{g}}{\rightarrow} G$. For any tangent vector $X_{a_{g}}^{*} \in T_{a_{g}}\left(T^{*}(G)\right)$, we recall the fundamental 1 -form $\theta$ at $a_{g} \in T^{*}(G)$ is given by

$$
\theta_{a}\left(X_{a}^{*}\right)=a_{g}\left(\pi_{*}\left(X_{a_{g}}^{*}\right)\right)
$$

and $\omega=d \theta$ defines the natural symplectic form on $T^{*}(G)$. Suppose $X^{*}$ is a leftinvariant vector field on $T^{*}(G)$, then $\pi_{*}\left(X_{a_{g}}^{*}\right)=L_{g^{*}}\left(\pi_{*}\left(X_{0_{e}}^{*}\right)\right)$ by the fact that $\pi$ commutes with left translations. $\pi_{*}\left(X_{a_{g}}^{*}\right)$ is independent of a so that $\pi_{*}\left(X_{a_{g}}^{*}\right)=$ $\pi_{*}\left(X_{\beta_{g}}^{*}\right)$ for all $\alpha, \beta \in g^{*}$. Hence $\pi_{*}\left(X^{*}\right)=X$ defines a left-invariant vector field on $G$. Suppose $c(s)$ is the one parameter subgroup of $T^{*}(G)$ generated by $X^{*}$, then $c(s)$ can be written as $c(s)=a(s)_{b(s)}$, where $a(s)$ is a curve in $g^{*}$ and $\pi(c(s))=b(s)$ is the one parameter subgroup of $G$ generated by $X$. In order to show $\omega$ is left-invariant on $T^{*}(G)$, it is sufficient to show

$$
\omega_{a_{g}}\left(X_{a_{g}}^{*}, Y_{a_{g}}^{*}\right)=\omega_{0}\left(X_{0}^{*}, Y_{0}^{*}\right)
$$

for any left-invariant vector fields $X^{*}$ and $Y^{*}$ on $T^{*}(G)$ and any $a_{8} \in T^{*}(G)$. To this end, let us compute $X^{*} \theta\left(Y^{*}\right)$ in the equation

$$
\begin{aligned}
\omega\left(X^{*}, Y^{*}\right) & =X^{*} \theta\left(Y^{*}\right)-Y^{*} \theta\left(X^{*}\right)-\theta\left(\left[X^{*}, Y^{*}\right]\right) . \\
X_{\boldsymbol{g}}^{*} \theta\left(Y^{*}\right) & =\lim _{s \rightarrow 0} \frac{1}{s}\left\{\left({ }^{t} \operatorname{Ad}\left(g^{-1}\right) a(s)\right)(Y)\right\}
\end{aligned}
$$

the value is independent of the choice of $\alpha$, hence especially for $g=e$,

$$
X_{0}^{*} \theta\left(Y^{*}\right)=\lim _{s \rightarrow 0} \frac{1}{s}\{a(s)(Y)\} .
$$

If we denote by $d(s)_{\exp s Y}$ the one parameter subgroup of $T^{*}(G)$ generated by $Y^{*}$, similar results are obtained for $Y^{*} \theta\left(X^{*}\right)$;

$$
Y_{a_{g}} \theta\left(X^{*}\right)=\lim _{s \rightarrow 0} \frac{1}{s}\left\{\left({ }^{t} A d\left(g^{-1}\right) d(s)\right)(X)\right\}
$$

and

Hence

$$
\dot{Y}_{0_{e}} \theta\left(X^{*}\right)=\lim _{s \rightarrow 0} \frac{1}{s}\{d(s)(X)\}
$$

$$
\begin{aligned}
\omega_{\boldsymbol{a}}\left(X_{a_{\boldsymbol{g}}}^{*}, Y_{a_{\boldsymbol{g}}}^{*}\right)= & \lim _{s \rightarrow 0} \frac{1}{s}\left\{\left({ }^{t} \operatorname{Ad}\left(g^{-1}\right) a(s)\right)(Y)\right\} \\
& -\lim _{s \rightarrow 0} \frac{1}{s}\left\{\left({ }^{t} \operatorname{Ad}\left(g^{-1}\right) d(s)\right)(X)\right\}-\alpha([X, Y])
\end{aligned}
$$


and

$$
\omega_{0}\left(X_{0}^{*}, Y_{0}^{*}\right)=\lim _{s \rightarrow 0} \frac{1}{s}\{a(s)(Y)\}-\lim _{s \rightarrow 0} \frac{1}{s}\{d(s)(X)\} .
$$

It is evident that the adjoint map is simply the identity map if $G$ is abelian and that the Lie algebra $g$ is also abelian. So $\omega_{a_{g}}\left(X_{a_{g}}^{*}, Y_{a_{g}}^{*}\right)=\omega_{0_{e}}\left(X_{0_{e}}^{*}, Y_{0_{e}}^{*}\right)$ shows $\omega$ is left-invariant. Conversely, if $\omega$ is left-invariant, we see that $\alpha([x, y])$ is the only term which does not involve $g$; so $\alpha([x, y])=0$ for all $\alpha \in g^{*}$ implies $[X, Y]=0$ for all $X, Y \in g$. That is to say $g$ is abelian and so is $G$.

In the following, we are going to show that the Lie algebra of a symplectic Lie group cannot be semisimple; but let us at first observe some interesting phenomena.

A Lie algebra $g$ is a vector space as well as an algebra with the multiplication satisfying $[x, y]=-[y, x]$ and Jacobi identity $[[x, y], z]+[[y, z], x]+$ $[[z, x], y]=0$. Let us denote by $V(g)$ the underlying vector space of $g$ and denote by $a$ an algebra whose underlying vector space is $V$. If we take, for instance, $g=g(n ; \mathbf{R})$, the Lie product in $g$ is given by $[x, y]=x \cdot y-y \cdot x$ where $x \cdot y$ is the matrix multiplication of $x$ and $y$ in $g h(n ; \mathbf{R})$. Then $V(3)$ is the vector space of $n \times n$ matrices and a can be taken as the associative algebra of $n \times n$ matrices whose underlying vector space is $V$. Furthermore, we see that $[x, y] \cdot z=x \cdot(y \cdot z)-y \cdot(x \cdot z)[13]$.

Definition. An algebra $a$ with underlying vector space $V(g)$ is said to be an algebra dominating the Lie algebra $g$ (or $g$ is subordinate to $a$ ) if the following two conditions are satisfied:

(i) $x \cdot y-y \cdot x=[x, y]$ for all $x, y \in V$,

(ii) for each $x \in V$, let $l(x)$ be the linear map of $V$ defined by $l(x) y=x \cdot y$; then $l$ is a representation of $g$ such that $l([x, y])=[l(x), l(y)]$.

Hence we may say that $g((n ; \mathbf{R})$ is subordinate to the associative algebra of all $n \times n$ matrices.

Theorem 6. Let $g$ be the Lie algebra of a symplectic Lie group G. Then $g$ is subordinate to an algebra $a$ and the multiplication in $a$ is defined by the symplectic form.

Proof. Suppose $\omega$ is a left-invariant symplectic form defined on $G$; since $\omega$ is of maximal rank, $i(x) \omega=0$ if and only if $x=0$ for $x \in$ g. Therefore, the map defined by $x \mapsto i(x) \omega$ is an isomorphism from the vector space $g$ to the vector space $g^{*}$ of left-invariant 1 -forms on $G$. Denote by $V$ the underlying vector space of g. Then for $x, y \in V, L_{x}(i(y) \omega) \in g^{*}$ so there is a unique $z \in V$ such that $i(z) \omega=L_{x}(i(y) \omega)$. It is easy to see the map $(x, y) \mapsto z$ is bilinear from $V \times V \rightarrow V$. We define $x \cdot y=z$. By this multiplication, an algebra with the underlying vector space $V$ is defined. Observing the fact that $i(y)\left(L_{x} \omega\right)=L_{y}(i(x) \omega)$, 
we have $i([x, y]) \omega=i(x, y) \omega-i(y \cdot x) \omega$ and $i([x, y] \cdot z) \omega=i(x \cdot(y \cdot z)) \omega-$ $i(y \cdot(x \cdot z))_{\omega}$, thus $[x, y]=x \cdot y-y \cdot x$ and $[x, y] \cdot z=x \cdot(y \cdot z)-y \cdot(x \cdot z)$. If $l$ is the map such that $l(x) y=x \cdot y$, then $l([x, y])=[l(x), l(y)]$. Hence $l$ defines a representation of $g$, and $g$ is subordinate to $a$.

Corollary. If a connected Lie group $G$ admits a two-sided invariant symplectic form $\omega$, then $G$ is abelian.

Proof. Since $\operatorname{Ad}(g) \omega=\omega$ for all $g \in G, L_{x} \omega=0$ for all $x \in g$. We see that $i(x \cdot y) \omega=i(x) L_{y} \omega=0$ implies $x \cdot y=0$ for all $x, y \in g$ and $[x, y]=0$. So $g$ is abelian.

Theorem 7. A semisimple Lie algebra is not subordinate to any algebra.

Proof. Assume otherwise that $g$ is the semisimple Lie algebra subordinate to an algebra $a$ and $l$ is the representation of $g$ in the underlying space $V$ of $g$ as well as of $a$. For $x \in g$, define $\rho(x)$ as an affine transformation of $V$ by $\rho(x)=l(x) v+x$ for all $v \in V$. We have $\rho([x, y])=[\rho(x), \rho(y)]$. Thus $\rho$ defines a representation of $g$ by infinitesimal affine transformations of $V$. Denote by $\epsilon$ the identity map of $V$ so that $\epsilon(x)=x$ for $x \in V$. $\epsilon$ is a $V$-valued 1-cochain in $C^{1}(g, l, V)$ and $\rho(x) v=l(x) v+\epsilon(x)$. We see $l(x) \epsilon(y)-l(y) \epsilon(x)-\epsilon([x, y])=0$. So $\epsilon$ is a 1 -cocycle in $Z^{1}(g, l, V)$ which coincides with $B^{1}(g, l, V)$ since $g$ is semisimple. Therefore there is $e \in V$ such that for any $x \in V, \epsilon(x)=l(x) e$ and $e$ is a right identity in $g$ such that $x=x \cdot e$. Furthermore, $\operatorname{ad}(e) x=[e, x]=$ $l(e) \cdot x-\epsilon(x)$, thus ad $(e)=l(e)-\epsilon$. Let $n$ be the dimension of $g$, and by taking traces of these maps of $V$, we have $\operatorname{Tr} \operatorname{ad}(e)=\operatorname{Tr} l(e)-n$. Since $g$ is semisimple, $\operatorname{Tr} \operatorname{ad}(e)=\operatorname{Tr} l(e)=0$. We are led to a contradiction and the theorem is proved.

Combining the above two theorems, we have achieved the following.

Theorem 8. Any semisimple Lie group has no left-invariant symplectic structure.

A Lie algebra 9 possesses a solvable ideal $x$ with the property that every solvable ideal of $g$ is contained in $x$; the ideal $x$ is called the solvable radical. The residue class algebra $g / x$ is semisimple. Let $\pi$ be the projection from $g$ onto $g / x$; then there exists a Lie algebra homomorphism $\rho: g / x \rightarrow g$ such that $\pi \circ \rho$ is the identity map on $g / x[5]$. We have the splitting exact sequence

$$
0 \rightarrow x \rightarrow g \rightarrow g / x \rightarrow 0
$$

and thus obtain a Levi decomposition $g=b+x$ (vector space sum) where $b$ is a semisimple subalgebra of $g$.

Theorem 9. A symplectic Lie group of dimension four has to be solvable. 
Proof. Assume $G$ is not solvable and whose Lie algebra has a Levi decomposition $g=8+x$. Since the dimension of a semisimple Lie algebra is at least three and $g$ itself cannot be semisimple, hence $\operatorname{dim} z=3$ and $\operatorname{dim} x=1$. Therefore it is evident that $\{$ is an ideal. Let $\omega$ be the symplectic structure, we also have $\omega(z, x)=0$. Because $x$ is one dimensional, $\omega(g, x)=0$ implies $x=\{0\}$. This is a contradiction.

If a Lie algebra can resolve into the direct sum of its solvable radical and a semisimple ideal, we have the following generalization.

Theorem 10. Suppose the Lie algebra $g$ of a symplectic group $G$ has a Levi composition $g=b+x$ as a Lie algebra direct sum; then $G$ is solvable.

Proof. Suppose $8 \neq\{0\}$ and $\omega$ is the symplectic structure. Since $\omega$ is closed, $\omega(z, x)=0$. Let $\left\{x_{1}, \cdots, x_{2 n}\right\}$ be a basis of $g$ such that $\left\{x_{1}, \cdots, x_{p}\right\}$ spans $\&$ and $\left\{x_{p+1}, \cdots, x_{2 n}\right\}$ spans $x$. The matrix of the skew-symmetric bilinear function $\omega$ on $g$ has

$$
\operatorname{det}\left(\omega\left(x_{i}, x_{j}\right)\right)=\operatorname{det}\left|\begin{array}{cc}
\omega\left(x_{i}, x_{j}\right) \\
1 \leq i, j \leq p
\end{array}\right| \operatorname{det}\left|\begin{array}{cc}
\omega\left(x_{i}, x_{j}\right) \\
p+1 \leq i, j \leq 2 n
\end{array}\right|
$$

By Theorem 8, the skew-symmetric matrix

$$
\left[\begin{array}{cc}
\omega\left(x_{i},\right. & \left.x_{j}\right) \\
1 \leq i, j \leq p
\end{array}\right]
$$

determined by the restriction of $\omega$ on $\{$ cannot be of maximal rank. Therefore $\omega$ cannot be of maximal rank on $g$ unless $s=\{0\}$ so that $G$ is solvable.

Corollary. A compact connected symplectic group is a torus.

Proof. Since $G$ is compact, the Lie algebra $g$ of $G$ has an invariant positive definite bilinear form, hence $g$ resolves into the direct sum of its center and a semisimple ideal [10] which has to be zero.

This is also an immediate consequence of the following theorem.

Theorem 11. Let $G$ be a connected symplectic Lie group of 2 dimensions. Suppose $H^{2 n}(g) \neq 0$; then $G$ is solvable.

Proof. Suppose the Lie algebra $g$ has a Levi decomposition $g=\underline{z}+\mathfrak{x}$ such that $\delta \neq\{0\}$. Let $g^{*}$ be the dual space of $g$ and let $\omega$ be a left-invariant symplectic form. Define a representation $\rho$ of $\{$ by the infinitesimal affine transformation of $g^{*}$ as follows:

$$
\begin{aligned}
\rho: & \left\lceil\rightarrow a\left(g^{*}\right),\right. \\
x & \mapsto L_{x}+i(x) \omega
\end{aligned}
$$


that is, for $\psi \in g^{*}, \rho(x) \psi=L_{x} \psi+i(x) \omega$. The linear part $L$ (Lie derivative) of $\rho$ is a representation of $\&$ by linear transformation of $g^{*}$ and the translation part $i$ ( ) $\omega$ of $\rho$ is a 1 -cocycle in $Z^{1}\left(\bar{b}, L, g^{*}\right)$. Since $H^{1}\left(8, L, g^{*}\right)=0, i() \omega$ is also a 1 -coboundary in $B^{1}\left(z L, g^{*}\right)$. Thus there exists $\theta \in g^{*}$ such that $L_{x} \theta=i(x)_{\omega}$ for all $x \in$ F. Since $L_{x} \theta=i(x) d \theta$, we have $i(x)(d \theta-\omega)=0$, so that $i(x)(d \theta-\omega)^{n}$ $=0$. Next we shall prove $\omega^{n}$ is cohomologous to zero in $C^{n}(g)$. For this purpose, let us choose a basis $\left\{x_{1}, \ldots, x_{r}, y_{1}, \ldots, y_{s} \mid r+s=2 n\right\}$ for $g$ such that $\left\{x_{1}, \ldots, x_{r}\right\}$ spans 8 and $\left\{y_{1}, \cdots, y_{s}\right\}$ spans $x$. Suppose $\left\{\xi^{1}, \cdots, \xi, \eta^{1}, \cdots, \eta^{s}\right\}$ is the dual basis in $g^{*}$. Then $(d \theta-\omega)^{n}=a \xi^{1} \wedge \ldots \wedge \xi^{\prime} \wedge \eta^{1} \wedge \cdots \wedge \eta^{s}$, where $a$ is a constant. A nonzero element $x$ of $\left\{\right.$ can be expressed as $x=b_{1} x_{1}+\cdots+b_{r} x_{r}$ such that not all of these constants $\left(b_{k}\right.$ 's) are zero. We have

$$
i(x)(d \theta-\omega)^{n}=\sum_{k=1}^{r} a(-1)^{k-1} b_{k} \xi^{1} \wedge \ldots \wedge \hat{\xi}_{k} \wedge \ldots \wedge \xi^{r} \wedge \eta^{1} \wedge \cdots \wedge \eta^{s}=0
$$

implies $a b_{k}=0$ for $1 \leq k \leq r$. Hence $a=0$ so that $(d \theta-\omega)^{n}=0$. That is to say the volume element $\omega^{n}$ of $G$ is an exact form which contradicts our assumption $H^{2 n}(g) \neq 0$. Therefore $\delta=\{0\}$ and $G$ is solvable.

The result was first obtained by Hano [4], but the method employed here is different from his.

A connected Lie group is said to be unimodular if det $\operatorname{Ad}(g)=1$ for all $g \epsilon$ $G$ or equivalently if $\operatorname{Tr}$ ad $(x)=0$ for all $x \in g$. An $n$-dimensional connected Lie group $G$ is unimodular if and only if $\operatorname{dim} H^{n}(g)=1$.

Corollary. A connected unimodular Lie group admitting a left invariant sym. plectic structure must be solvable.

Hano has proved that a connected nilpotent Lie group admitting a left invariant Kählerian structure must be abelian. However, this is not the case for symplectic Lie groups, as can be seen in the following example. Therefore, we may say that not all symplectic Lie groups are given by Lie groups with leftinvariant Kählerian structures.

Example 1. Take

$$
G=\left\{\left[\begin{array}{cccc}
e^{t} & 0 & 0 & 0 \\
0 & 1 & x & z \\
0 & 0 & 1 & y \\
0 & 0 & 0 & 1
\end{array}\right] \mid t, x, y, z \in \mathbf{R}\right\}
$$


Evidently, $g$ is nilpotent. We may choose a basis $x_{1}, x_{2}, x_{3}$ and $x_{4}$ for $g$ such that $x_{1}$ and $x_{4}$ are in the center and $\left[x_{2}, x_{3}\right]=x_{4}$, and define a skew-symmetric bilinear function $\omega$ of maximal rank whose matrix form with respect to the chosen basis is

$$
\left(\omega\left(x_{i}, x_{j}\right)\right)=\left[\begin{array}{rrrr}
0 & 0 & 1 & 0 \\
0 & 0 & 0 & 1 \\
-1 & 0 & 0 & 0 \\
0 & -1 & 0 & 0
\end{array}\right] .
$$

By straightforward verification, $d \omega=0$. Let $\Gamma$ be a discrete subgroup of $G$ consisting of

$$
\left[\begin{array}{cccc}
e^{n_{1}} & 0 & 0 & 0 \\
0 & 1 & n_{2} & n_{4} \\
0 & 0 & 1 & n_{3} \\
0 & 0 & 0 & 1
\end{array}\right]
$$

where $n_{i}$ 's are integers. The set of left residue classes $\Gamma \backslash G$ gives a compact symplectic manifold.

There is a natural Kählerian structure defined on the upper half complex plane $H$, and the group $G=\left\{\left[\begin{array}{ll}x & y \\ 0 & 1\end{array}\right] \mid x>0, y \in \mathbf{R}\right\}$ acts on $H$ transitively. Hence the Kählerian structure induces a left-invariant symplectic structure on $G$. Combining this fact and Theorem 9, we may say that all symplectic groups of dimension less than or equal to four are solvable. However, it is not true for the case of dimension six.

Example 2. Let $g$ be the Lie subalgebra of the Lie algebra $g \mathrm{l}(3 ; \mathbf{R})$ consisting of all the matrices of the form

$$
\left[\begin{array}{ccc}
a_{1} & a_{2} & a_{3} \\
0 & a_{4} & a_{5} \\
0 & a_{6} & -a_{4}
\end{array}\right] .
$$

Then $g$ contains a three-dimensional simple Lie subalgebra. Let $\left\{A_{i} \mid 1 \leq i \leq 6\right\}$ be a basis of $g$, whose entry corresponding to their subscripts is 1 and zero elsewhere. On $g$, there is a maximal rank 2 -cocycle $\omega$ whose matrix form with respect to the chosen basis is 


$$
\left(\omega\left(A_{i}, A_{j}\right)\right)=\left[\begin{array}{rrrrrr}
0 & 1 & 0 & 0 & 0 & 0 \\
-1 & 0 & 0 & 1 & 0 & 0 \\
0 & 0 & 0 & 0 & 0 & 1 \\
0 & -1 & 0 & 0 & -1 & 0 \\
0 & 0 & 0 & 1 & 0 & 0 \\
0 & 0 & -1 & 0 & 0 & 0
\end{array}\right] .
$$

Hence $\omega$ induces an invariant symplectic structure on the connected Lie group with Lie algebra $g$ in $G L(3 ; \mathbf{R})$.

\section{BIBLIOGRAPHY}

1. C. Chevalley, Theory of Lie groups. I, Princeton Math. Series, vol. 8, Princeton Univ. Press, Princeton, N. J., 1946. MR 7, 412.

2. - Théorie des groupes de Lie. II. Groupes algèbres, Actualités Sci. Indust., no. 1152, Hermann, Paris, 1951. MR 14, 448.

3. C. Chevalley and Samuel Eilenberg, Cohomology theory of Lie groups and Lie algebras, Trans. Amer. Math. Soc. 63 (1948), 85-124. MR 9, 567.

4. Jun-ichi Hano, On Kaehlerian homogeneous spaces of unimodular Lie groups, Amer. J. Math. 79 (1957), 885-900. MR $20 \# 2477$.

5. G. Hochschild, The structure of Lie groups, Holden-Day, San Francisco, Calif., 1965. MR $34 \# 7696$.

6. S. Kobayashi and K. Nomizu, Foundations of differential geometry. Vol I, Interscience, New York, 1963. MR 27 \#2945.

7. B. Kostant, Quantization and unitary representations. I. Prequantization, Lectures in Modern Analysis and Applications. III, Lecture Notes in Math., vol. 170, Springer, Berlin, 1970, pp. 87-208. MR 45 \#3638.

8. Yozo Matsushima, Differential geometry, Dekker, New York, 1972.

9. Richard S. Palais, A global formulation of the Lie theory of transformation groups, Mem. Amer. Math. Soc. No. 22 (1957). MR 22 \#12162.

10. L. S. Pontrjagin, Continuous groups, 2nd ed., GITTL, Moscow, 1954; English transl., Gordon and Breach, New York, 1966. MR 17, 171; 34 \#1439.

11. Shlomo Sternberg, Lectures on differential geometry, Prentice-Hall, Englewood Cliffs, N. J., 1964. MR 33 \#1797.

12. J.-M. Souriau, Structure des systèmes dynamiques, Maîtrises de mathématiques, Dunod, Paris, 1970. MR 41 \#4866.

13. E. B. Vinberg, The theory of convex homogeneous cones, Trudy Moskov. Mat. Obšč. 12 (1963), 303-358 = Trans. Moscow Math. Soc. 1963, 340-403. MR 28 \#1637.

DEP ARTMENT OF MATHEMATICS, UNIVERSITY OF NOTRE DAME, NOTRE DAME, INDIANA 64556

Current address: Department of Mathematics, St. Edward's University, Austin, Texas 78704 